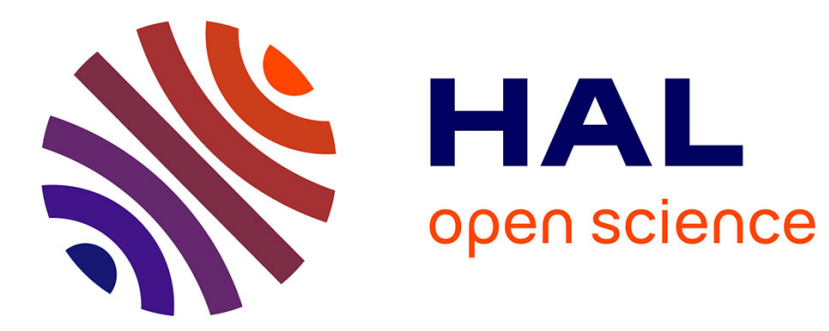

\title{
On modal probability and belief
}

Andreas Herzig, Dominique Longin

\section{To cite this version:}

Andreas Herzig, Dominique Longin. On modal probability and belief. European Conference on Symbolic and Quantitative Approaches to Reasoning with Uncertainty (ECSQARU 2003), Jul 2003, Aalborg, Denmark. pp.62-73, 10.1007/b11792 . hal-03534094

\section{HAL Id: hal-03534094 https://hal.science/hal-03534094}

Submitted on 19 Jan 2022

HAL is a multi-disciplinary open access archive for the deposit and dissemination of scientific research documents, whether they are published or not. The documents may come from teaching and research institutions in France or abroad, or from public or private research centers.
L'archive ouverte pluridisciplinaire HAL, est destinée au dépôt et à la diffusion de documents scientifiques de niveau recherche, publiés ou non, émanant des établissements d'enseignement et de recherche français ou étrangers, des laboratoires publics ou privés. 


\title{
On Modal Probability and Belief
}

\author{
Andreas Herzig and Dominique Longin \\ Institut de Recherche en Informatique de Toulouse (CNRS - UMR 5505) \\ 118 route de Narbonne, F-31062 Toulouse CEDEx 04, France \\ \{herzig, longin\}@irit.fr \\ www.irit.fr/ Andreas.Herzig \\ www.irit.fr/ Dominique. Longin
}

\begin{abstract}
We investigate a simple modal logic of probability with a unary modal operator expressing that a proposition is more probable than its negation. Such an operator is not closed under conjunction, and its modal logic is therefore non-normal. Within this framework we study the relation of probability with other modal concepts: belief and action.
\end{abstract}

\section{Introduction}

Several researchers have investigated modal logics of probability. Some have added probability measures to possible worlds semantics, most prominently Fagin, Halpern and colleagues [1]. They use modal operators of knowledge, $\mathcal{K} \phi$ expressing that the agent knows that $\phi$, and they introduce modal operators of the kind $w(\phi) \geq b$ expressing that "according to the agent, formula $\phi$ holds with probability at least $b$ ".

Others have studied the properties of comparative probability, following Kraft, Pratt, and Seidenberg, and Segerberg. They use a relation $\phi>\psi$ (that can also be viewed as a binary modal construction) expressing " $\phi$ is more probable than $\psi "$.

Only few have studied a still more qualitative notion, viz. the modal logic of constructions of the kind $\mathcal{P} \phi$ expressing that $\phi$ is more probable than $\neg \phi$ (or at least as probable as $\neg \phi$ ). Among those are Hamblin [2], Burgess [3], and T. Fine [4]. Halpern and colleagues have studied the similar notion of likelihood $[5,6]$. Also related is research on modal logics allowing to count the accessible worlds $[7,8]$. One can also read $\mathcal{P} \phi$ as "probability of $\phi$ is high", and interpret "high" as "greater than $b$ ", for $1>b \geq 0.5$. We then basically get the same account as for $b=0 . .^{1}$

\footnotetext{
${ }^{1}$ As suggested by one of the reviewers, another option is to interpret $\mathcal{P} \phi$ not as a two-valued modal proposition but as a many-valued modal proposition, as it is done in [9, Chapter 8] and [10]. There, the truth degree of $\mathcal{P} \phi$ is taken as $\operatorname{Prob}(\phi)$, so the bigger is the probability of $\phi$, the 'more true' is the proposition $\mathcal{P} \phi$. One can then express that $\phi$ is more probable for the agent than $\neg \phi$ by a non-classical implication $\mathcal{P} \neg \phi \rightarrow \mathcal{P} \phi$.
} 
Probably one of the reasons for the lack of interest in such approaches is that the corresponding logical systems are very poor, and do not allow to obtain completeness results w.r.t. the underlying probability measures. ${ }^{2}$

We here investigate the logic of the modal operator $\mathcal{P}$. We start by analyzing its properties, in particular in what concerns the interplay with the notion of belief. Contrarily to comparative possibility, such an operator is not closed under conjunction, and therefore its modal logic is non-normal [14].

We then turn to semantics. While probability distributions over sets of accessible worlds are helpful to explain modal constructions such as $\mathcal{P} \phi$, it is known that they do not allow complete axiomatizations. We here study a semantics that is closer to the set of properties that we have put forward. Our models are minimal models in the sense of [14], that are based on neighborhood functions instead of probability distributions. The logic is a non-normal, monotonic modal logic. ${ }^{3}$

Within this framework our aim is to study the relation of probability with other modal concepts such as belief and action. We propose principles for the interplay between action, belief, and probability, and formulate successor state axioms for both belief and probability. While there is a lot of work on probabilistic accounts of belief and action, as far as we are aware there is no similar work relating modal probability to belief and action.

\section{Preliminaries}

For the time being we do not consider interactions between several agents, and therefore we only consider a single agent.

\subsection{Atomic Formulas, Atomic Actions}

We have a set of atomic formulas $A t m=\{p, q, \ldots\}$. Our running example will be in terms of playing dice; we thus consider atomic formulas $d_{1}, d_{2}, \ldots$, respectively expressing "the dice shows 1", etc.

We have set of atomic actions $A c t=\{\alpha, \beta, \ldots\}$. In our example we have the throw action of throwing the dice, and the actions observe $_{1}$, observe $_{2}, \ldots$ of the agent observing that the dice shows 1 , etc.

Actions are not necessarily executed by the agent under concern, but may be executed by other agents or by nature. (So we might as well speak about events instead of actions.)

We could have considered complex actions, but for the sake of simplicity we shall not do so here.

\footnotetext{
${ }^{2}$ Note that things are simpler if we do not take probability theory but possibility theory: As shown in [11,12], Lewis' operator of comparative possibility [13] provides a complete axiomatization of qualitative possibility relations.

${ }^{3}$ Hence our semantics is rather far away from probabilities. This might be felt to be at odds with intuitions, but as a matter of fact what we have done is to exactly capture all that can be formally said about the property $\mathcal{P}$ of being probable.
} 
¿From these ingredients complex formulas will be built together with modal operators in the standard way.

\subsection{Modal Operators}

We have a standard doxastic modal operator $\mathcal{B}$, and the formula $\mathcal{B} \phi$ is read "the agent believes that $\phi$ ", or " $\phi$ is true for the agent". For example $\neg \mathcal{B} d_{6}$ expresses that the agent does not believe that some dice shows "6". The formula $\mathcal{B}\left(d_{1} \vee d_{2} \vee d_{3} \vee d_{4} \vee d_{5} \vee d_{6}\right)$ expresses that the agent believes the dice shows one of $1,2,3,4,5$, or 6 .

Moreover we have a modal operator $\mathcal{P}$ where $\mathcal{P} \phi$ is read " $\phi$ is probable for the agent". The dual $\neg \mathcal{P} \neg \phi$ expresses that $\phi$ is not improbable. (This operator has been considered primitive in some papers in the literature.) For example, $\mathcal{P}\left(d_{1} \vee d_{2} \vee d_{3} \vee d_{4}\right)$ expresses that it is probable for the agent that the dice shows one of $1,2,3$, or 4 . $\neg \mathcal{P} d_{6}$ expresses that it is improbable for the agent that the dice shows " 6 ".

Finally, for every action $\alpha \in$ Act we have a dynamic logic operator $[\alpha]$. The formula $[\alpha] \phi$ is read " $\phi$ holds after every execution of $\alpha$ ". For example $\neg[$ throw $] \neg d_{6}$ expresses that the dice may show 6 after the throwing action. $\neg \mathcal{P}$ [throw $] d_{6}$ expresses that this is improbable for the agent. [throw] $\mathcal{P} \neg d_{6}$ expresses that after throwing the dice it is probable for the agent that it did not fall 6. [throw] $\left[\right.$ observe $\left._{6}\right] \mathcal{B} d_{6}$ expresses that after throwing the dice and observing that it fell 6 the agent believes that it fell 6 .

\subsection{Relations Agreeing with a Probability Measure}

$\mathcal{P}$ can also be viewed as a relation on formulas. Let Prob be any subjective probability measure defined on formulas that is associated to the agent. When it holds that

$$
\mathcal{P} \phi \text { iff } \operatorname{Prob}(\phi)>\operatorname{Prob}(\neg \phi)
$$

we say that $\mathcal{P}$ agrees with Prob.

\subsection{Hypotheses about Action}

We make some hypotheses about actions and their perception by the agent. They permit to simplify the theory.

Public Action Occurrences. We suppose that the agent perceives action occurrences completely and correctly. For example whenever a dice is thrown the agent is aware of that, and whenever the agent believes a dice is thrown then indeed such an action has occurred. (One might imagine that action occurrences are publicly announced to all agents.) 
Public Action Laws. We suppose that the agent knows the laws governing the actions. Hence the agent knows that after throwing a dice the effect always is that $1,2,3,4,5$, or 6 show up, and that 1 and 2 cannot show up simultaneously, etc.

Non-informativity. We suppose that all actions are non-informative. Noninformative actions are actions which are not observed by the agent beyond their mere occurrence. In particular the agent does not observe the outcome of nondeterministic actions such as that of throwing a dice. Upon learning that such an action has occurred the agent updates his belief state: he computes the new belief state from the previous belief state and his knowledge about the action laws. Hence the new belief state neither depends on the state of the world before the action occurrence, nor on the state of the world after the action occurrence.

In our example we suppose that the throw action is non informative: the agent throws the dice without observing the outcome. If the agent learns that the action of throwing a dice has been executed then he does not learn which side shows up.

Clearly, the action observe of observing the outcome of the throw action is informative: the new belief state depends on the position of the dice in the real world. Other examples of informative actions are that of looking up a phone number, testing if a proposition is true, telling whether a proposition is true, etc.

Nevertheless, the agent is not disconnected from the world: he may learn that some proposition is true (i.e. that some action of observing that some proposition has some value has occurred). For example, when he learns that it has been observed that the dice fell 6 (i.e. he learns that the action of observing 6 has been executed) then he is able to update his belief state accordingly. Indeed, the observe $_{i}$ actions are non-informative according to our definition: when the agent learns that observe $_{i}$ has occurred then he is able to update his belief state accordingly, and there is no need to further observation of the world. Other examples of noninformative actions are that of learning that the phone number of another agent is $N$, testing that a proposition is true (in the sense of Dynamic Logic tests), telling that a proposition is true, etc.

\section{Axioms for Probability}

In this section we give an axiomatization for $\mathcal{P}$.

The inference rule for $\mathcal{P}$ is

$$
\text { if } \phi \rightarrow \psi \text { then } \mathcal{P} \phi \rightarrow \mathcal{P} \psi
$$

and the axioms are as follows:

$$
\begin{gathered}
\mathcal{P} \top \\
\mathcal{P} \phi \rightarrow \neg \mathcal{P} \neg \phi
\end{gathered}
$$


These axioms match those that have been put forward in the literature, e.g. those in [4]. As stated there, it seems that there are no other principles of probability that could be formulated using $\mathcal{P}$.

Clearly such an axiomatization is sound w.r.t. the intended reading:

Theorem 1. Let Prob be any probability measure, and suppose the property $\mathcal{P}$ agrees with $\mathcal{P}$, i.e. $\mathcal{P} \phi$ iff $\operatorname{Prob}(\phi)>\operatorname{Prob}(\neg \phi)$. Then $\mathcal{P}$ satisfies $\left(R M_{\mathcal{P}}\right),\left(N_{\mathcal{P}}\right)$, $\left(D_{\mathcal{P}}\right)$.

Another way of expressing this is that whenever we define $\mathcal{P} \phi$ by $\operatorname{Prob}(\phi)>0.5$ then $\mathcal{P}$ satisfies $\left(\mathrm{RM}_{\mathcal{P}}\right),\left(\mathrm{N}_{\mathcal{P}}\right),\left(\mathrm{D}_{\mathcal{P}}\right){ }^{4}$

Nevertheless, such an axiomatics is not complete w.r.t. probability measures. This will be illustrated in Section 9 .

\section{Axioms for Belief}

Following [15] we suppose a standard KD45 axiomatics for $\mathcal{B}$ : we have the inference rule

$$
\text { if } \phi \rightarrow \psi \text { then } \mathcal{B} \phi \rightarrow \mathcal{B} \psi
$$

and the following axioms:

$$
\begin{gathered}
\mathcal{B} \top \\
\mathcal{B} \phi \rightarrow \neg \mathcal{B} \neg \phi \\
(\mathcal{B} \phi \wedge \mathcal{B} \psi) \rightarrow \mathcal{B}(\phi \wedge \psi) \\
\mathcal{B} \phi \rightarrow \mathcal{B B} \phi \\
\neg \mathcal{B} \phi \rightarrow \mathcal{B} \neg \mathcal{B} \phi
\end{gathered}
$$

Hence the set of beliefs is closed under logical consequences, and we suppose agents are aware of their beliefs and disbeliefs, i.e. we suppose introspection.

\section{Axioms Relating Belief and Probability}

What is the relation between $\mathcal{P}$ and $\mathcal{B}$ ? According to our reading we should have that things that are believed are also probable for an agent, i.e. we expect $\mathcal{B} \phi \rightarrow \mathcal{P} \phi$ to hold. The following main axiom will allow us to derive that:

$$
(\mathcal{B} \phi \wedge \mathcal{P} \psi) \rightarrow \mathcal{P}(\phi \wedge \psi)
$$

Just as for the case of beliefs and disbeliefs, agents are aware of probabilities. This is expressed by the following two axioms:

$$
\begin{aligned}
\mathcal{P} \phi & \rightarrow \mathcal{B P} \phi \\
\neg \mathcal{P} \phi & \rightarrow \mathcal{B} \neg \mathcal{P} \phi
\end{aligned}
$$

Other principles of introspection for $\mathcal{P}$ will be derived from them in the sequel.

\footnotetext{
${ }^{4}$ This can be strengthened: if for some $b>0.5, \mathcal{P} \phi$ is defined as $\operatorname{Prob}(\phi)>b$ then $\mathcal{P}$ satisfies $\left(\mathrm{RM}_{\mathcal{P}}\right),\left(\mathrm{N}_{\mathcal{P}}\right),\left(\mathrm{D}_{\mathcal{P}}\right)$. Note that thus our axioms do not conflict with the view of $\mathcal{P} \phi$ as "probability of $\phi$ is high".
} 


\subsection{Some Provable Formulas}

1. if $\vdash \phi \equiv \psi$ then $\vdash \mathcal{P} \phi \equiv \mathcal{P} \psi$ This can be derived from $\left(\mathrm{RM}_{\mathcal{P}}\right)$.

2. $\vdash \neg(\mathcal{P} \phi \wedge \mathcal{P} \neg \phi)$

This is an equivalent formulation of $\left(\mathrm{D}_{\mathcal{P}}\right)$.

3. $\vdash \neg \mathcal{P} \perp$ By $\left(\mathrm{D}_{\mathcal{P}}\right), \mathcal{P} \perp \rightarrow \neg \mathcal{P} \neg \perp$. Then $\mathcal{P} \perp \rightarrow \perp$ by $\left(\mathrm{N}_{\mathcal{P}}\right)$.

4. $\vdash \mathcal{B} \phi \rightarrow \mathcal{P} \phi$

This follows from $\left(\mathrm{N}_{\mathcal{P}}\right)$ and (C-MIX), putting $\psi=\top$.

5. $\vdash(\mathcal{B} \phi \wedge \neg \mathcal{P} \neg \psi) \rightarrow \neg \mathcal{P} \neg(\phi \wedge \psi)$

From (C-MIX) together with $\left(\mathrm{RM}_{\mathcal{P}}\right)$ it follows $\vdash(\mathcal{B} \phi \wedge \mathcal{P} \neg(\phi \wedge \psi) \rightarrow \mathcal{P} \neg \psi)$.

6. $\vdash \mathcal{P} \phi \rightarrow \neg \mathcal{B} \neg \phi$

This follows from the next formula.

7. $\vdash(\mathcal{P} \phi \wedge \mathcal{P} \psi) \rightarrow \neg \mathcal{B} \neg(\phi \wedge \psi)$

This can be proved as follows: first, (C-MIX) together with $\left(\mathrm{RM}_{\mathcal{P}}\right)$ entails $\vdash(\mathcal{P} \phi \wedge \mathcal{B} \neg(\phi \wedge \psi)) \rightarrow \mathcal{P} \neg \psi$. Then with (D) we get $\vdash(\mathcal{P} \phi \wedge \mathcal{B} \neg(\phi \wedge \psi)) \rightarrow$ $\neg \mathcal{P} \psi$, from which the theorem follows by classical logic.

8. $\vdash(\mathcal{B}(\phi \rightarrow \psi) \wedge \mathcal{P} \phi) \rightarrow \mathcal{P} \psi$

This follows from (C-MIX) together with $\left(\mathrm{RM}_{\mathcal{P}}\right)$.

9. $\vdash \mathcal{P} \phi \equiv \mathcal{B P} \phi$

The " $\rightarrow$ " direction follows from (4-MIX). The other direction follows from $\left(5\right.$-MIX) and $\left(\mathrm{D}_{\mathcal{P}}\right)$.

10. $\vdash \neg \mathcal{P} \phi \equiv \mathcal{B} \neg \mathcal{P} \phi$

The " $\rightarrow$ " direction follows from (5-MIX). The other direction follows from $(4-\mathrm{MIX})$ and $\left(\mathrm{D}_{\mathcal{P}}\right)$.

11. $\vdash \mathcal{P} \phi \equiv \mathcal{P} \mathcal{P} \phi$

The " $\rightarrow$ " direction follows from (4-MIX). The other direction follows from $\left(5\right.$-MIX) and $\left(\mathrm{D}_{\mathcal{P}}\right)$.

12. $\vdash \neg \mathcal{P} \phi \equiv \mathcal{P} \neg \mathcal{P} \phi$

The " $\rightarrow$ " direction follows from (5-MIX) and $\vdash \mathcal{B} \phi \rightarrow \mathcal{P} \phi$. The other direction follows from (4-MIX) and $\left(\mathrm{D}_{\mathcal{P}}\right)$.

13. $\mathcal{P} \mathcal{B} \phi \rightarrow \mathcal{P} \phi$

From $\vdash \mathcal{B} \phi \rightarrow \mathcal{P} \phi$ it follows that $\mathcal{P B} \phi \rightarrow \mathcal{P} \mathcal{P} \phi$. And as we have seen, $\mathcal{P P} \phi \rightarrow \mathcal{P} \phi$.

\subsection{Some Formulas that Cannot Be Proved}

The following formulas will not be valid in our semantics. Non-deducibility will follow from soundness.

1. $\mathcal{P} \phi \rightarrow \mathcal{B} \phi$

This would in fact identify $\mathcal{P}$ and $\mathcal{B}$.

2. $\mathcal{P} \phi \rightarrow \mathcal{P B} \phi$

Indeed, given that we expect $\mathcal{P} \phi \wedge \neg \mathcal{B} \phi$ to be consistent, such a formula would even lead to inconsistency (due to axioms $\left(5_{\mathcal{B}}\right)$ and (C-MIX). 
3. $(\mathcal{P} \phi \wedge \mathcal{P} \psi) \rightarrow \mathcal{P}(\phi \wedge \psi)$

This would clash with the probabilistic intuitions: $\operatorname{Prob}(\phi)>\operatorname{Prob}(\neg \phi)$ and $\operatorname{Prob}(\psi)>\operatorname{Prob}(\neg \psi)$ does not imply $\operatorname{Prob}(\phi \wedge \psi)>\operatorname{Prob}(\neg(\phi \wedge \psi))$.

4. $(\mathcal{P} \phi \wedge \mathcal{P}(\phi \rightarrow \psi)) \rightarrow \mathcal{P} \psi$

The reasons are the same as for the preceding formula.

\section{Axioms for Action}

We suppose the logic of action is just $\mathrm{K}$. We therefore have the inference rule

$$
\text { if } \phi \rightarrow \psi \text { then }[\alpha] \phi \rightarrow[\alpha] \psi
$$

and the following axioms:

$$
\begin{gathered}
{[\alpha]^{\top}} \\
([\alpha] \phi \wedge[\alpha] \psi) \rightarrow[\alpha](\phi \wedge \psi)
\end{gathered}
$$

Hence our logic of action is a simple version of dynamic logic [16].

\section{Axioms Relating Belief and Action}

We recall that we have stated in Section 2.4

- that the agent perceives action occurrences completely and correctly,

- that he knows the laws governing the actions, and

- that actions are non-informative, i.e. the agent does not learn about particular effects of actions beyond what is stipulated in the action laws.

As action effects are not observed, when the agent learns that the action of throwing a dice has been executed then he does not learn whether it fell 6 or not.

In $[17,18]$ we have argued that under these hypotheses the following axioms of "no forgetting" (NF) and "no learning" (NL) are plausible. They express that the agent's new belief state only depends on the previous belief state and the action whose occurrence he has learned.

$$
\begin{gathered}
(\neg[\alpha] \perp \wedge[\alpha] \mathcal{B} \phi) \rightarrow \mathcal{B}[\alpha] \phi \\
(\neg \mathcal{B}[\alpha] \perp \wedge \mathcal{B}[\alpha] \phi) \rightarrow[\alpha] \mathcal{B} \phi
\end{gathered}
$$

For the "no learning" axiom, we must suppose that the action $\alpha$ is executable (else from $[\alpha] \mathcal{B} \phi$ ) we could not deduce anything relevant). Similarly, for the "no forgetting" axiom we must suppose that the agent does not believe $\alpha$ to be inexecutable (else from $\mathcal{B}[\alpha] \phi$ we could not deduce anything relevant). When the agent believes $\alpha$ to be inexecutable and nevertheless learns that it has occurred then he must revise his beliefs. In $[19,18]$ it has been studied how AGM style belief revision operations [20] can be integrated. We do not go into details here, and just note that both solutions can be added in a modular way. 
$\left(\mathrm{NF}_{\mathcal{B}}\right)$ and $\left(\mathrm{NL}_{\mathcal{B}}\right)$ together are equivalent to

$$
(\neg[\alpha] \perp \wedge \neg \mathcal{B}[\alpha] \perp) \rightarrow([\alpha] \mathcal{B} \phi \equiv \mathcal{B}[\alpha] \phi)
$$

Axioms having this form have been called successor state axioms in cognitive robotics, and it has been shown that (at least in the case of deterministic actions) they enable a proof technique called regression [21,22].

\section{Axioms Relating Probability and Action}

Suppose before you learn that a dice has been thrown it is probable for the agent that the dice will not fall $6: \mathcal{P}[$ throw $] \neg d_{6}$. When the agent learns that the dice-throwing action has been executed (without learning the outcome, cf. our hypotheses) then it is probable for him that the dice does not show 6 . Therefore the following no-learning axiom for $\mathcal{P}$ is plausible for non-informative actions:

$$
(\neg[\alpha] \perp \wedge[\alpha] \mathcal{P} \phi) \rightarrow \mathcal{P}[\alpha] \phi
$$

The other way round, when it is probable for the agent that 6 shows up after throw then (as we have supposed that he does not observe the outcome of throwing) it was already probable for the agent that 6 would show up before learning that the action has been executed. This is expressed by the following no-forgetting axiom.

$$
(\neg \mathcal{P}[\alpha] \perp \wedge \mathcal{P}[\alpha] \phi) \rightarrow[\alpha] \mathcal{P} \phi
$$

Again, both axioms are conditioned by executability of $\alpha$ (respectively belief of executability of $\alpha$ ).

\section{Semantics}

Actions are interpreted as transition systems: truth of a formula $[a l p h a] \phi$ in a state (alias possible world) means truth of $\phi$ in all states possibly resulting from the execution of $\alpha$.

Truth of the formula $\mathcal{B} \phi$ means truth of $\phi$ in all worlds that are possible for the agent.

In what concerns the formula $\mathcal{P} \phi$, the intuition is that to every possible world there is associated a probability measure over the set of epistemically accessible worlds, and that $\operatorname{Prob}(\phi)>\operatorname{Prob}(\neg \phi)$. Sometimes the intuition is put forward that among the set of accessible worlds there are more worlds where $\phi$ is true than worlds where $\phi$ is false. We shall show in the sequel that such an explanation is misleading.

A frame is a tuple $\left\langle W, B, P,\left\{R_{\alpha}: \alpha \in A c t\right\}\right\rangle$ such that 
- $W$ is a nonempty set of possible worlds

$-B: W \longrightarrow 2^{W}$ maps worlds to sets of worlds

$-P: W \longrightarrow 2^{2^{W}}$ maps worlds to sets of sets of worlds

$-R_{\alpha}: W \longrightarrow 2^{W}$ maps worlds to sets of worlds, for every $\alpha \in$ Act

Thus for every possible world $w \in W, B(w)$ and $R_{\alpha}(w)$ are sets of accessible worlds as usual.

By convention, for a set of possible worlds $V \subseteq W$ we suppose $R_{\alpha}(V)=$ $\bigcup_{v \in V} R_{\alpha}(v)$, etc.

$P(w)$ is a set of sets possible worlds. Although intuitively $P$ collects 'big' subsets of $B$ (in the sense that for $V \in P, V$ contains more elements than its complement w.r.t. $W, W \backslash V)$, there is no formal requirement reflecting this.

Every frame must satisfy some constraints: for every $w \in W$,

$\left(\mathrm{d}_{\mathcal{B}}\right) \quad B(w) \neq \emptyset$

$\left(45_{\mathcal{B}}\right) \quad$ if $w^{\prime} \in B(w)$ then $B\left(w^{\prime}\right)=B(w)$

$\left(\mathrm{n}_{\mathcal{P}}\right) \quad P(w) \neq \emptyset$

$\left(\mathrm{d}_{\mathcal{P}}\right) \quad$ if $V_{1}, V_{2} \in P(w), V_{1} \cap V_{2} \neq \emptyset$

(c-mix) if $V \in P(w)$ then $V \subseteq B(w)$

(45-mix) if $w \in B(w)$ then $P\left(w^{\prime}\right)=P(w)$

$\left(\right.$ nf-nl $\left.l_{\mathcal{B}}\right) \quad$ if $w^{\prime} \in R_{\alpha}(w)$ and $R_{\alpha}(B(w)) \neq \emptyset$ then $B\left(w^{\prime}\right)=R_{\alpha}(B(w))$.

$\left(\right.$ nf-nl $\left.\mathcal{P}_{\mathcal{P}}\right)$ if $w^{\prime} \in R_{\alpha}(w)$ then $P\left(w^{\prime}\right)=\left\{R_{\alpha}(V): V \in P(w)\right.$ and $\left.R_{\alpha}(V) \neq \emptyset\right\}$

As usual a model is a frame together with a valuation: $\mathcal{M}=\langle\mathcal{F}, V\rangle$, where $V: A t m \longrightarrow 2^{W}$ maps every atom to the set of worlds where it is true. To formulate the truth conditions we use the following abbreviation:

$$
\|\phi\|_{\mathcal{M}}=\{w \in W: \mathcal{M}, w \models \phi\}
$$

Then given a model $\mathcal{M}$, the truth conditions are as usual for the operators of classical logic, plus:

$-\mathcal{M}, w \models \phi$ if $\phi \in A t m$ and $w \in V(\phi)$

$-\mathcal{M}, w \models \mathcal{B} \phi$ if $B(w) \subseteq\|\phi\|_{\mathcal{M}}$

- $\mathcal{M}, w \models \mathcal{P} \phi$ if there is $V \in P(w)$ such that $V \subseteq\|\phi\|_{\mathcal{M}}$

$-\mathcal{M}, w \models[\alpha] \phi$ if $R_{\alpha}(w) \subseteq\|\phi\|_{\mathcal{M}}$

\subsection{An Example}

Let us give an example. It will at the same time illustrate that the intuition of $P(w)$ 'collecting more than $50 \%$ of the accessible worlds' is misleading.

Let the agent learn in $w_{0}$ that a dice has been thrown. Then we might suppose that after throw the situation is described by a possible world $w$ where $B(w)=\left\{v_{1}, \ldots, v_{6}\right\}$ such that $v_{i} \in V\left(d_{j}\right)$ iff $i=j$, and where $P(w)$ is the set of all subsets of $B(w)$ containing more than half of the worlds in $B(w)$, i.e. $P(w)=\{V \subseteq B(w): \operatorname{card}(V)>3\}$.

Now suppose we are in a game where a player is entitled to throw his dice a second time if (and only if) his first throw was a 6 . Let throwif6 describe that 
deterministic conditional action. We have thus $R_{\text {throwif6 }}\left(v_{6}\right)=\left\{v_{6_{1}}^{\prime}, \ldots, v_{6_{6}}^{\prime}\right\}$ with $v_{6_{i}}^{\prime} \in V\left(d_{j}\right)$ iff $i=j$. For $i \leq 5$, we have $R_{\text {throwif }}\left(v_{i}\right)=\left\{v_{i}^{\prime}\right\}$ with $v_{i}^{\prime} \in V\left(d_{j}\right)$ iff $v_{i} \in V\left(d_{j}\right)$. According to our semantics, the situation after a completed turn can be described by a possible world $w^{\prime}$ where

$-R_{\text {throwifg }}(w)=\left\{w^{\prime}\right\}$

- $B\left(w^{\prime}\right)=R_{\text {throwif6 }}\left(v_{6}\right) \cup \bigcup_{i \leq 5} R_{\text {throwif6 }}\left(v_{i}\right)$.

- The neighborhood $P\left(w^{\prime}\right)$ of $w^{\prime}$ contains in particular $\left\{v_{1}^{\prime}, v_{2}^{\prime}, v_{3}^{\prime}, v_{4}^{\prime}\right\}$, although this set contains much less than half of the worlds in $B\left(w^{\prime}\right)$.

\subsection{Soundness and Completeness}

Our axiomatization is sound w.r.t. the present neighborhood semantics:

Theorem 2. If $\phi$ is provable from our axioms and inference rules, then $\phi$ is valid in neighborhood semantics.

We conjecture that we have completeness, too. The only nonstandard part of the Henkin proof concerns the neighborhood semantics: In principle, for all $w \in W$ and $V \in P(w)$ our axiom (C-MIX) only enforces that there is some $V^{\prime} \in P(w)$ such that $V^{\prime} \subseteq V \cap B(w)$. What we would like our model to satisfy is that $V \in B(w)$. In order to guarantee that frames must be transformed in the following way:

Lemma 1. Let $\langle\mathcal{F}, V\rangle$ be any model satisfying all the constraints except (cmix). If $\mathcal{F} \models(C-M I X)$ and $\langle\mathcal{F}, V\rangle, w \models \phi$ then there is a model $\left\langle\mathcal{F}^{\prime}, V^{\prime}\right\rangle$ such that $\left\langle\mathcal{F}^{\prime}, V^{\prime}\right\rangle$ satisfies the constraints and such that $\left\langle\mathcal{F}^{\prime}, V^{\prime}\right\rangle, w \models \phi$.

Proof. We define $W^{\prime}=W, V^{\prime}=V, B^{\prime}=B, R_{\alpha}^{\prime}=R_{\alpha}$, and $P^{\prime}(w)=\{V \in$ $P(w): V \subseteq B(w)\}$ As for every $V \in P(w)$ there is some $V^{\prime} \in P(w)$ such that $V^{\prime} \subseteq V \cap B(w), P(w)$ is nonempty. Moreover, we can prove by induction that for every $w \in W$ and every formula $\psi$, we have $\langle\mathcal{F}, V\rangle, w \models \psi$ iff $\left\langle\mathcal{F}^{\prime}, V^{\prime}\right\rangle, w \models \psi$.

\subsection{The Relation with Probability Measures}

In any case, our neighborhood semantics differs from the standard semantics in terms of probability measures. The latter is not complete w.r.t. probability measures, as announced in Section 3.

Theorem $3([4])$. : Let Atm $=\{a, b, c, d, e, f, g\}$. Take a model $\mathcal{M}$ where

$$
\begin{aligned}
& \text { - W }=2^{\text {Atm }} \\
& \text { - for every } w \in W, N(w)=\{\text { efg, abg, adf, bde, ace, cdg, bcf }\} \text {, where efg is } \\
& \quad \text { used to denote }\{e, f, g\}, \text { etc. } \\
& -V(p)=\{w \in W: p \in W\}
\end{aligned}
$$

Then $\mathcal{M}$ satisfies the above constraints on neighborhood frames, but their is no agreeing probability measure. 


\section{Conclusion}

We have investigated a 'very qualitative' notion of probability, that of a formula being more probable than its negation. We have presented the axioms governing its relation with belief, and we have proposed principles for the interplay between action, belief, and probability.

While there is a lot of work on probabilistic accounts of belief and action, as far as we are aware there is no similar work relating modal probability to belief and action.

While we provide a probabilistic account of belief, we do not consider probabilistic action here. Therefore (and just as in the logics of belief and action) uncertainty can only diminish as actions occur, and on the long run probabilities will converge towards belief, in the sense that we will have $P(w)=\{B(w)\}$. Just as in the case of shrinking belief states, this is unsatisfactory. In future work we shall introduce misperception (as already done for beliefs in [18]) and probabilistic actions in order to improve the account.

\section{Acknowledgements}

Thanks to the three reviewers, all of which have provided comments that hopefully enabled us to improve our exposition.

\section{References}

1. Fagin, R., Halpern, J.Y.: Reasoning about knowledge and probability. Journal of the ACM 41 (1994) 340-367

2. Hamblin, C.: The modal 'probably'. Mind 68 (1959) 234-240

3. Burgess, J.P.: Probability logic. J. of Symbolic Logic 34 (1969) 264-274

4. Walley, P., Fine, T.L.: Varieties of modal (classificatory) and comparative probability. Synthese 41 (1979) 321-374

5. Halpern, J., Rabin, M.: A logic to reason about likelihood. Artificial Intelligence J. 32 (1987) 379-405

6. Halpern, J., McAllester, D.: Likelihood, probability, and knowledge. Computational Intelligence $\mathbf{5}$ (1989) 151-160

7. Fattorosi-Barnaba, M., de Caro, F.: Graded modalities I. Studia Logica 44 (1985) $197-221$

8. van der Hoek, W.: On the semantics of graded modalities. J. of Applied Nonclassical Logics (JANCL) 2 (1992)

9. Hajek, P.: Metamathematics of fuzzy logic. Kluwer (1998)

10. Godo, L., Hajek, P., Esteva, F.: A fuzzy modal logic for belief functions. In: Proc. 17th Int. Joint Conf. on Artificial Intelligence (IJCAI'01). (2001) 723-732

11. Farias del Cerro, L., Herzig, A.: A modal analysis of possibility theory. In: Proc. European Conf. on Symbolic and Quantitative Approaches to Uncertainty (ECSQAU'91). Number 548 in LNCS, Springer Verlag (1991) 58-62 (short version; long version published in FAIR'91). 
12. Farias del Cerro, L., Herzig, A.: A modal analysis of possibility theory (invited paper). In Jorrand, P., Kelemen, J., eds.: Proc. of the Int. Workshop on Foundations of AI Research (FAIR 91). Number 535 in LNAI, Springer Verlag (1991) 11-18 (short version published in ECSQAU'91).

13. Lewis, D.: Counterfactuals. Basil Blackwell, Oxford (1973)

14. Chellas, B.: Modal logic: An introduction. Cambridge University Press (1980)

15. Hintikka, J.K.K.: Knowledge and belief. Cornell University Press, Ithaca, N.Y. (1962)

16. Harel, D.: Dynamic logic. In Gabbay, D.M., Günthner, F., eds.: Handbook of Philosophical Logic. Volume II. D. Reidel, Dordrecht (1984) 497-604

17. Herzig, A., Lang, J., Longin, D., Polacsek, T.: A logic for planning under partial observability. In: Proc. Nat. (US) Conf. on Artificial Intelligence (AAAI'2000), Austin, Texas (2000)

18. Herzig, A., Longin, D.: Sensing and revision in a modal logic of belief and action. In van Harmelen, F., ed.: Proc. ECAI2002, IOS Press (2002) 307-311

19. Shapiro, S., Pagnucco, M., Lespérance, Y., Levesque, H.J.: Iterated belief change in the situation calculus. In: Proc. KR2000. (2000) 527-538

20. Gärdenfors, P.: Knowledge in Flux: Modeling the Dynamics of Epistemic States. MIT Press (1988)

21. Reiter, R.: The frame problem in the situation calculus: A simple solution (sometimes) and a completeness result for goal regression. In Lifschitz, V., ed.: Artificial Intelligence and Mathematical Theory of Computation: Papers in Honor of John McCarthy. Academic Press, San Diego, CA (1991) 359-380

22. Levesque, H.J., Reiter, R., Lesprance, Y., Lin, F., Scherl, R.: GOLOG: A logic programming language for dynamic domains. J. of Logic Programming (1997) special issue on reasoning about action and change. 